\title{
An Extensive Research Survey on Networked Control Systems (NCS)
}

\author{
Anupama S. \\ Assistant Professor, Department of Electronics \& Communication Engineering, \\ Vidya Vikas Institute of Engineering \& Technology, Mysore, \\ Karnataka, India
}

\begin{abstract}
The system which closes its control loops with the help of communication networks is known as networked control systems (NCSs). These communication networks include both the feedback and control signals exchanged among the system components like controller, sensors, actuators, etc. The NCSs exhibits its applicability in the wide range of applications in signal processing and industrial control. Recently, the NCS has become an interesting research topic for both academia and industry. With the advancement in the research trend, there is a need for consolidation of the latest information and knowledge on that to understand the research requirements. Thus, this paper introduces a research survey on the different types of approaches evolved for NCS in recent years. This paper is initialized by discussing the essential aspects of the NCS and its applications.
\end{abstract}

Further, the NCS caused issues are described and then followed with the discussion on different controlling mechanisms. Also, to find the gaps in the current research some of the recent research surveyed. Followingly, the current state of the art in the research of NCS is given. Finally, the paper is ended-up with a conclusion and futuristic scope of the research survey.

\section{Keywords}

Actuators, Controllers, NCS, Network induced delay, packet dropouts, Sampling, Sensors.

\section{INTRODUCTION}

A system consisting control loops are closed by a communication network where both the feedback as well as control signals incorporated (exchange) with system components like sensor, actuators, and controllers (such systems are referred as networked control systems (NCSc)). The significant feature of the NCS is that it links both the physical space and cyberspace so that multiple operations can be achieved remotely. Hence, the NCS exhibits many advantages over the traditional control systems. Also, the NCS does the elimination of the unnecessary wiring among the system components that directly minimizes the complexity as well as overall system design cost and implementation cost subjected to the controller system [1]. The redesigning and modification in the NCS design is very simple where any addition and removal of the system component can be performed more significantly without inducing many changes in the system architecture of NCS. Thus, NCSs are considered for various application areas like industry control (i.e., vehicle industry, automobile wiring by adapting networked control techniques). The successful use of NCS is applied in the human surveillance system and other process control systems like water treatment $[2,3]$. But the use of communication networks mainly induces some critical issues. An example of such an issue is periodic sampling having high frequency leads to the intrusion of heavy load into the network system. This load may cause the network congestion, limited (bit rate) of communication causing network generates packet dropouts and delays [4], quantization errors takes place due to quantization of sampled signals [4]. These critical issues are occurred due to communication networks are mainly referred as sources of system performance degradation or destabilization of NCS; also some significant impacts of the network generated delays on NCS are given [4]. As the concept of the NCS exists between communication principle and control principle, the NCSs analysis is more complicated and is gaining more attention. The last decade has witnessed various significant researches are introduced towards NCS. Author Zhang et al. [5], 2016 given a concept of resilient estimation for NCS which yields reliable communication ability. In 2017, Lee et al. [6] presented an NCS with asynchronous quantization, sampling for both the channels of transmission and receiver. Similarly, Pasand and Montazeri [7] discussed the structural and scheduling property for NCS along with limitations like bandwidth and transmission delay. In 2018 , the work on periodic event-triggered sampling and dual rate control for NCS are witnessed which can be used in unmanned aerial vehicle (UAV) (Cuenca et al. [8]) and work on packed based dual rate by using proportional-integralderivative (PID) for NCS having slow rate sensing (by Cuenca et al. [9]). Nowadays, the NCS is moving towards distributed NCSs that are multi-disciplinary mechanisms aiming to generate the network component and architectures which are capable of incorporating distributed sensors, actuators, and control algorithms over a communication network for applicability in real-time applications [10].

This paper introduces an extensive research survey on the current state of the art in the NCS domain. The paper is outlined with subsections like essentials of NCS (section 2), different control approaches (Section 3), review of existing researches in NCS (Section 4), Research gaps identified (Section 5), state of the art in current research work (Section 6 ). Finally, the conclusive points of the survey paper are given with futuristic scope of it are discussed in Section 7.

\section{ESSENTIALS OF NETWORK CONTROL SYSTEMS}

In the communication networking system, control system plays a crucial role that responsible for handling, command and regulates the all other interrelated devices or a system like, e.g., sensors, actuators, controllers, etc. In NCS, the control loops will be closed via network communication channel, which can be transferred with other correlated devices outside the network control system. Figure.1 illustrates the graphical view of the network control system 
which is connected with cyber-space to physical components so that it can easily share the several tasks by remotely.

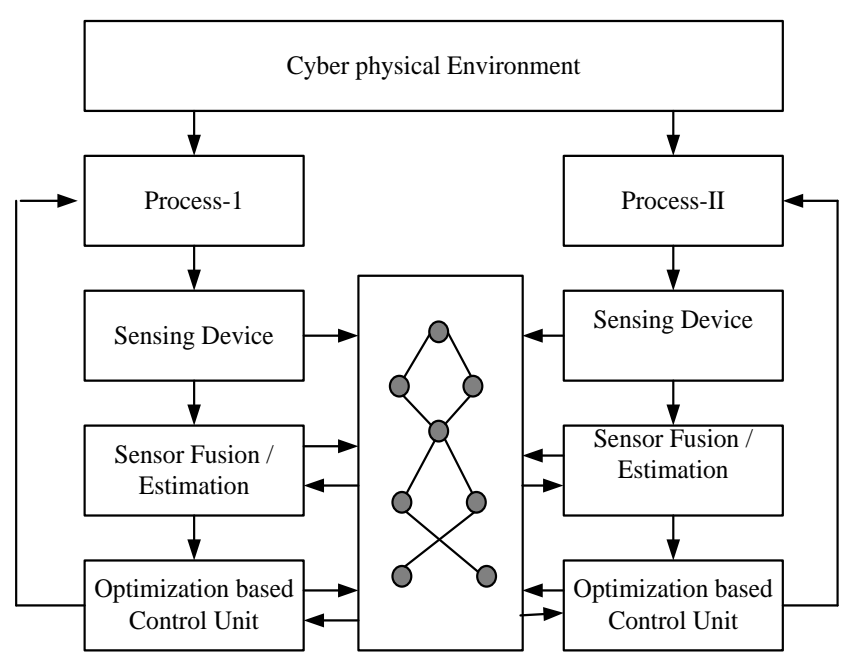

Fig 1: Model for Network Control Systems

Communication signals among control system components for several processes are transformed via communication network channel. The modern network controlling systems are largely equipped with several modules where signals are shared with communication channels, and computation carries out either continuously or periodically. Modern theory, algorithm designs, and demonstration must be introduced in which the fundamental input or output information (signals) are data packets which may travel at defined times, not fixed in the order.

However, the network control system architecture separates the conventional elements of sensing, fusion or estimation, controllers and an actuator for a given module across the network and allows the communication link among the systems [11]. This framework can be utilized for either a single system design or multiple system design which interacts via the network. These systems are widely found in applicatory areas like industry control. Example, in-vehicle industries, an extensive wiring automobile model is utilized by introducing network controlling systems; additionally, these control systems are successfully applied in surveillance systems [12] and various engineering systems like as in "Waster Treatment Process." There exist several problems caused by NCS, i.e., 1) sampling, 2) network delay, 3) data packet dropouts and 4) quantization errors, which are explained in the following.

\subsection{Sampling}

Instantaneous signals required to be sampled before the information shared via the communication network. There are two ways to sample the instantaneous signals; 1) time triggered and 2) event triggered sampling.

The time-triggered sampling is also referred to as "Riemann Sampling" which characterizes the signals when an event is triggered, i.e., next time sampling instance occurs after the elapse of the fixed period. The periodic sampling is significantly utilized in traditional digital control-systems because design and analysis of such kind of sampling process may easier. Whereas an event triggered sampling is the level crossing sampling which shows the way an event is triggered, i.e., next sampling occurs because of sample signal changes with the precise amount. This type of signal sampling is the natural process where human behaves like a control system. Additionally, it may contribute to majorly reduction of traffic loads. Over the last decades, the increasing attention is more paid towards event triggered for network control systems [13]-[14].

\subsection{Network caused Delay}

In communication control systems, network caused delays may occur through different factors, (e.g., less bandwidth, traffic loads and communication protocols. Generally, there are two different types of network delays; 1) sensor controller delays and 2) actuator controller delays.

The sensor controller delays define the period from the instant when a signal is sampled with instant during the generation of corresponding control signals; whereas; actuator controller delays refers how long a signal is forwarded from control system to actuator. Therefore, the network caused delays strongly based on network variable conditions; they are and random but upper bounded. As a result, network caused delays are designed as an intervening time-varying delay and "Markov chain" with known signal transition probabilities, along with partly known signal transition probability [15] [16].

Nevertheless, for some control systems, like an offshore platform and D-Van oscillator, network caused delays might have positive results on system performance. For example: in, B. Zhang and Han proposed a dynamic network-based model of the offshore platform also introduced a networkbased feedback control scheme [17].

\subsection{Data Packet Dropouts}

The most prominent issue caused by the communication control system is the packet dropouts (PD). Since of limited bandwidth, data packets might be dropped out during the transmission process. There are two kinds of packet dropouts; 1) Network caused PD and 2) Active PD.

Typically, network caused PD occurs during the network condition is worst, i.e., if the network traffic is overloaded, an event of data transmission timeouts and data transmission errors in a communication network, etc. Since in all these cases packet dropout will happen by communication network itself. Therefore, this type of packet dropouts is called as network caused PD.

While in active packet dropouts, data packet forwarded earlier and arrives at the destination later, packet disordering will happen. Though, packet disordering can be transformed eventually in some reliable transmission protocols, e.g., TCP. In and presented some effective methods, like logical $\mathrm{ZOH}$ scheme and message rejection scheme which carry out the active packet dropouts [18].

\section{DIFFERENT CONTROLLING MECHANISM}

The recent decade has witnessed research on control of networked control systems (NCSs) by considering the abovementioned issues.

\subsection{Control of Sample Data (SD)}

If the control components exhibit in the same place, then NCS can be considered as digital control systems. The mechanism of SD control was given in the mid of 20th century. In Mikheev et al. [19], a control mechanism of input delay (ID) 
is introduced for sample data that has gained popularity due to development of linear matrix inequality method and brought effectiveness in network control mechanisms. The use of ID mechanism helps to obtain a maximum upper bound of two samplings and respective controllers. The last decade has witnessed significant advancement in sampled data control by using the ID mechanism. In addition to the ID mechanism, various other control and analysis methods were introduced. The SD system under consideration is modelled as a continuous time system. However, stability analysis is performed by using a discrete time Lyapunov theorem in Seuret et al. [20]. Similarly, an impulsive approach is presented in Naghshtabrizi et al. [21] to analyse the stability of SD systems by using a discontinuous Lyapunov functional (LF). Further, the works of Chen and Zheng [22] have improved the mechanism in which LF is continuous at response time. Also, a model of continuous time delay system is introduced in Gao et al. [23] with a stochastic parameter satisfying Bernoulli distribution for SD systems.

\subsection{Networked control}

The NCCs faces challenges like Network injecting delays and packet dropouts. Considering these issues, various methods were developed for stability analysis and control design for NCCs. Some of the mechanisms are highlighted below.

\subsection{Time Delay System Mechanism}

This mechanism is developed by considering the ID mechanism of SD systems (as in Yue et al. [24]). Considering the time delay system mechanism, a closed loop can be designed as a system having a time-varying delay. The data on the sensor controller delay, actuators delay, and packet dropouts are implicitly considered an upper bound of ID.

\subsection{Markovian Jumping System Mechanism}

This mechanism is designed for control and analysis of NCCs which is subjected to discrete time domain (as in Seiler and Sengupta [25]). The network caused interruption or delay is considered and developed a closed loop NCS as a Markovian jumping system and used an LQG optimal controller design mechanism.

\subsection{Switched System Mechanism}

This mechanism with arbitrary switching is utilized to analyze and control NCSs. Similarly, various research tries like Zhang and $\mathrm{Yu}$ [26], Donkers et al. [27], Hetel et al. [28], Kruszewski et al. [29], Lin and Antsaklis [30], Sun et al. [31], etc., are modelled by using arbitrary switching mechanism. Among these techniques, few are continuous and discrete time domain approaches.

\subsection{Stochastic system mechanism}

The packet dropouts and network caused delays may have a random characteristic in networked control systems (as in Nilsson et al. [32]). A stochastic system mechanism is an efficient tool that helps in analyzing and controlling NCSs with random network injecting delays and packet dropouts. Some of the approaches are found in Yang et al. [33], Donkers et al. [34] and Tabbar and Nesic et al. [35].

\subsection{Event-Triggered Control}

This mechanism, the control of executed tasks is determined by event occurrences than elapse of fix time-triggered control. The advantages discussed in the work of Arzen [36], where it is found that event triggered control can lead to the large reduction in resources usage with the slight decrement in the performance enhancement. By considering these advantages, a systematic event-based design of stabilizing feedback control principle is introduced in Tabuada [37]. As the event triggered control has attained a lot of attention in NCSs (as in Lunze and Lehmann [38], Wang and Lemmon [39]).

\section{REVIEW OF LITERATURE}

The use of wireless network systems has gained the majority of interest by all industries and organizations. The adoption of wireless communication technology makes industries able to implement new visions for analyzing new system architectures for controlling process. It has also been seen that system design and architecture analysis suffers from the various problems due to the nonlinear nature of industrial control processes. Most of the researchers focus on improving and optimizing the stability of Networked Control System. Therefore, this section presents the existing work that is carried for analyzing the currents progress in the domain of Networked Control System (NCS).

A study on Nonlinear NCS has been carried out by the Wu et al. [40], in which the author has investigated a problem related with the adaptive fuzzy control for single-input-single-output with network delay and data loss. Afterward, a fuzzy logic system and novel adaptive control system is used to optimize the network delay and data loss in the transmission process. Furthermore, two examples are adopted in the experiment for analyzing the effectiveness and efficiency of the presented system by considering delay and data loss in transmission.

Similarly, the work of $\mathrm{Xu}$ et al. [41], have considered the problem associated with existing optimal control of nonlinearNCS due to network uncertainties and imperfection such as packet loss and network caused delay. In order to alleviate this problem, the author presents a novel scheme based on the time-dependent neuro-dynamic programming. The introduced schemes utilize neural network technique, critic neural network, actor neural network, and Lyapunov theory in order to achieve hypothetical efficient control management system for nonlinear-NCS in the presence of uncertain constraints due to network imperfection. The experimental outcomes of study deliver that the presented scheme achieves effective performance under in the presence of uncertainties of NCS.

The study of Qiu et al. [42], have carried a survey on the fuzzy logic system based nonlinear NCS with considering various networks caused constraints such as data loss, transmission delay, signal quantization, and packet delivery delay. The author has highlighted some essential complexities and issues of analyzing and designing fuzzy-model-based nonlinear NCS. According to the finding of [42], the author has tried to attract the attention of researchers toward the resolving the issues of fuzzy control systems in their future work.

In the same way, another work carried out by Khanesar et al. [43], concentrated on the issue of time-varying network delay and packet loss and presents a novel sliding controller model based on the Pade approximation function and fuzzy logic system to handle the transmission delay and packet loss problem. Also, the author has also used a Lyapunov function to ensure adopted mechanisms stability. The outcome of this study displays that the presented sliding controller has very good ability to control the nonlinear and dynamical systems over a network in the presence of external disturbance, timevarying transmission delay, and data loss. 
In [44], Yuan and $\mathrm{Wu}$ present an advanced control design model for NCS by considering two networks caused delay that is measurement delay \& actuation delay. The presented model is constructed with IQC framework coupled with impulse response function. The experimental outcome demonstrates that the presented model achieves effective performance after applied to the DC motor system.

An advance and strong adaptive controller based on the fuzzy system for NCS is presented by Hamdy et al. [45], to cope with the delay and packet drop problem caused by the nonlinear system. The presented controller has a filter error that is used to handle the delay issue in the NCS. Additionally, the authors have used a Lyapunov function to validate the efficiency of the presented system. The experimental and simulated results display that the presented control system achieves a good result for controlling packet loss and ensures the boundness of all signals in the closed loop with good tracking and regulation.

The work carried out by Jahromi and Seyedi [46], have offered an innovative design approach based on the Lyapunov direct method for stabilizing the time-invariant NCS. A set of stability conditions is formulated using the Lyapunov function for designing a light algorithm that gives disperse observer controller network that stands for the global asymptotic stability.

The work of $\mathrm{Li}$ et al. [47], have addressed the issue of robust tracking control of NCSwith network caused delay, packet dropouts, and packet and external disturbance. The author has presented a novel networked predictive control algorithm based on k-order adaptive discrete-time sliding- mode control. Moreover, the presented model is designed uses the estimated disturbance as weight factors and synthesizes the slidingmode values of all $\mathrm{k}$ times, to enhance the robustness of tracking control. In the experimental task, a DC motor servo system is utilized for system analysis.

The work carried out by $\mathrm{Wu}$ et al. [48], presents a novel control approach for trigger control in discrete-time NCS settings for the packet loss problem. By using the dwell-time mechanism, the author developed possible appropriate criteria to achieve closed-loop system stability. The presented approach is applied to the DC motor system for an experiment which shows that it obtains effective performance to control the packet loss in the transmission process.

It has also found that quantization often degrades NCS performance and stability. To mitigate the impacts of quantization the effort of Almakhles et al. [49], have designed an adaptive Quantizer model for industrial NCS. The model uses a sliding mode controller to estimate the inputs of the quantizer and to reduce the effects of quantization with data loss by encoding the signals by 1 bit per samples. The experiments were performed on a DC motor application, and in addition, a quantized feedback controller is designed to control the DC motor position. The Simulation outcome reveals that the presented model achieves effective property that reduces the data rate, quantization noise, and increase system performances.

An observer-based framework is constructed in which a stabilization problem in discrete-time NCS is considered by the Li et al. [50] in which the authors have put their effort to control the time delay problem and data losses issues in T-S fuzzy systems. A new stabilizing controller is developed to provide a separation principle to NCS. The experimental effects show that the presented approach obtains better result after using performing a mathematical analysis on two examples that are networked nonlinear system and networked mass-spring system.

A novel approach of delay distribution is adopted in the study of Tang et al. [51], for continuous-time NCS with timevarying delay transmission. Also, indicator function and advanced Lyapunov-Krasovskii technique are used for realtime distribution process for input delays, so that delaydistribution based necessary conditions can be extracted for the deterministic closed- loop enhanced stability. Furthermore, two numerical examples are used to evaluate the effectiveness and advantages of the presented approach.

The work of Lu et al. [52], have investigated fuzzy filtering stability and design problem for NCS with network generated delay and quantization. Further, the event-based triggered model and the quantizer is designed to improve the network stability and stable resources utilization with efficient network bandwidth. For analyzing the effectiveness of proposed model two numerical examples are used which shows that it is very effective for improving NCS imperfection and is use-full for wireless communication and industrial control applications.

Tang et al. [53] have presented a fuzzy model based strong design approach for a nonlinear NCS with time-varying transmission delay and an interval based joint approach of delay-distribution with enhanced Lyapunov-Krasovskii mechanism for real-time distribution of input delays. Further, three numerical examples such as $2 \mathrm{D}$ nonlinear damper mechanical system, 4D joint robot arm system and 2D rigid robot system with external disturbance are taken for analyzing the effectiveness of the proposed system.

Analysing the system at its initial design phase is essential to avoid the system failure in its active duration. Mo et al. [54] presented a linear discrete-time based novel stochastic model to control the packets dropouts, transmission delay and to improve the network reliability. Here in this, an example of an industrial heat exchanger NCS is used to illustrate the ability of presented design. The experimental effects of the presented approach display that it has great potential to mitigate the issue of network reliability in the nonlinear NCS.

Wang and Liu [55] investigated the problem that arises in the stability of NCS with delay time factor and data packet dropout at the transmission process. The author built a discrete-time switched NCS framework through which the exponential stability of the networked system can be obtained to handle packet dropout and latency. By improving network performance, ensuring security is also a crucial factor in the NCS.

The work of Sa et al. [56], presents a secure system named as random switching controller to counter the passive attacks. The simulation of the proposed method is very effective for tackling various types of passive attacks against NCS.

Feng et al. [57] have focused on the problem of fault-tolerant against actuator fault for the uncertain NCS. The author uses a Markov chain to model time delay factor and state augmentation method is used to design closed-loop system and further designed a feedback controller system for ensuring the stability of resultant closed loop system. The experimental result demonstrates that the presented approach can resolve the problem associated with actuator fault. 
A 100 meter Ethernet Switched is used in the work of Ming Li [58] to solve the problem caused due to the delay factor and to ensure that no packet loss in the data transmission process. From the experimental viewpoint, the delay caused by the single $100 \mathrm{~m}$ Ethernet is less than other class of NCS, and the performance of the presented approach is similar to non-NCS. So it is beneficial to apply a single $100 \mathrm{M}$ Ethernet Switched to all types of control system.

The work of Saha et al. [59] has offered a dynamic scheduling technique for designing resisting model for handling a group of controllers within the presence of network delay and distributed computational resources. The authors have suggested that the presented model is useful in the incorporated architecture of NCS. The performance of the proposed model is demonstrated through an example of some inverted pendulums system. The Experimental effects display that the presented technique efficiently reduces the packet drop rates.

To avoid quality degradation factor Zhiwen and Hongtao [60] have designed a framework in which LQR technique is used to analyze the network and network bandwidth is considered as energy in order to eliminate the problem of network congestion. The author focused on enhancing the quality of network performance by improving the network quality of services. Later the author has used a closed-loop scheduling technique for bandwidth management.

Another work of Yang et al. [61] studied the problem related to the control process in NCS under Denial of service (DoS) attack. The Author has introduced intrusion detection system based on game theory approach in network layer to defend the DoS attacks in NCS and to obtain efficient package delivery. Also, an iterative adaptive optimization algorithm is used in the control layer to resolve the problem of control with actuator saturation. The performance of the presented approach is achieved by the performing two numerical examples which shows the presented model is efficient to resist the DoS attacks and improve the control process in the NCS actuator.

Li et al. [62] have studied the issue related to communication and control co-design mechanism for linear NCS. A slotted ALOHA protocol is used to resolve the channel access issues so that sensor can share the resource of wireless technology. Moreover, a feedback controller based on pole assignment technique and convergent descent optimization algorithm is used to obtain guaranteed stability and good delivery of data packets in NCS.

The work of Wang and $\mathrm{Yu}$ et al. [63] presents active quantization scheme based on a Lyapunov-krasovskii functional approach for nonlinear uncertain NCS. The objective of the proposed system is to enhance the zooming-in algorithm to convey quick and improved closed-loop system convergence. The experimental outcome delivers that the presented approach achieves better convergence property compared to existing related works. The summary of the above-stated literature is given in Table.1.

Table 1: Summary of Existing Works

\begin{tabular}{|l|l|l|l|}
\hline Author & Problem & $\begin{array}{l}\text { Solution } \\
\text { Approach }\end{array}$ & Advantages \\
\hline
\end{tabular}

\begin{tabular}{|c|c|c|c|}
\hline $\begin{array}{l}\text { Wu et al. } \\
\text { [40] }\end{array}$ & $\begin{array}{l}\text { Network } \\
\text { delay and } \\
\text { data loss }\end{array}$ & $\begin{array}{l}\text { A fuzzy logic } \\
\text { system based } \\
\text { novel } \\
\text { adaptive } \\
\text { controller } \\
\text { system }\end{array}$ & $\begin{array}{l}\text { Reduces } \\
\text { network } \\
\text { delay and } \\
\text { data loss }\end{array}$ \\
\hline $\begin{array}{l}\text { Xu et al. } \\
\text { [41] }\end{array}$ & $\begin{array}{l}\text { Control } \\
\text { problem in } \\
\text { nonlinear } \\
\text { NCS }\end{array}$ & $\begin{array}{l}\text { neuro- } \\
\text { dynamic } \\
\text { programming, } \\
\text { critic neural } \\
\text { network, actor } \\
\text { neural } \\
\text { network, and } \\
\text { Lyapunov } \\
\text { theory }\end{array}$ & $\begin{array}{l}\text { Improves } \\
\text { the control } \\
\text { process and } \\
\text { reduces } \\
\text { network } \\
\text { congestion }\end{array}$ \\
\hline $\begin{array}{l}\text { Qiu et al. } \\
\text { [42] }\end{array}$ & $\begin{array}{l}\text { Data loss, } \\
\text { transmission } \\
\text { delay, signal } \\
\text { quantization, } \\
\text { and packet } \\
\text { delivery } \\
\text { delay }\end{array}$ & $\begin{array}{l}\text { Investigated } \\
\text { fuzzy logic } \\
\text { system based } \\
\text { nonlinear } \\
\text { NCS }\end{array}$ & $\begin{array}{l}\text { Highlighted } \\
\text { essential } \\
\text { point for } \\
\text { designing } \\
\text { fuzzy-model } \\
\text { based } \\
\text { nonlinear } \\
\text { NCS }\end{array}$ \\
\hline $\begin{array}{l}\text { Khanesar } \\
\text { et al. } \\
{[43]}\end{array}$ & $\begin{array}{l}\text { Time- } \\
\text { varying } \\
\text { network } \\
\text { delay and } \\
\text { packet loss }\end{array}$ & $\begin{array}{l}\text { Pade } \\
\text { approximation } \\
\text { function and } \\
\text { fuzzy logic } \\
\text { system }\end{array}$ & $\begin{array}{l}\text { Controls } \\
\text { data loss } \\
\text { and delay in } \\
\text { nonlinear } \\
\text { systems }\end{array}$ \\
\hline $\begin{array}{l}\text { Yuan } \\
\text { and } \mathrm{Wu} \\
{[44]}\end{array}$ & $\begin{array}{l}\text { Measurement } \\
\text { delay \& } \\
\text { actuation } \\
\text { delay }\end{array}$ & $\begin{array}{l}\text { Control } \\
\text { design model } \\
\text { with IQC } \\
\text { framework } \\
\text { coupled and } \\
\text { impulse } \\
\text { response } \\
\text { function }\end{array}$ & $\begin{array}{l}\text { Reduces } \\
\text { delay factor } \\
\text { and } \\
\text { increases } \\
\text { good packet } \\
\text { delivery } \\
\text { ratio }\end{array}$ \\
\hline $\begin{array}{l}\text { Hamdy } \\
\text { et al. } \\
{[45]}\end{array}$ & $\begin{array}{l}\text { delay and } \\
\text { packet drop } \\
\text { in nonlinear } \\
\text { NCS }\end{array}$ & $\begin{array}{l}\text { Robust } \\
\text { adaptive } \\
\text { controller } \\
\text { based fuzzy } \\
\text { system and } \\
\text { Lyapunov } \\
\text { function }\end{array}$ & $\begin{array}{l}\text { Effective } \\
\text { for } \\
\text { controlling } \\
\text { packet loss }\end{array}$ \\
\hline $\begin{array}{l}\text { Jahromi } \\
\text { and } \\
\text { Seyedi } \\
{[46]}\end{array}$ & $\begin{array}{l}\text { Time- } \\
\text { invariant } \\
\text { Uncertain } \\
\text { NCS }\end{array}$ & $\begin{array}{l}\text { Stabilizing } \\
\text { approach } \\
\text { based } \\
\text { Lyapunov } \\
\text { function }\end{array}$ & $\begin{array}{l}\text { Makes } \\
\text { uncertain } \\
\text { NCS stable }\end{array}$ \\
\hline $\begin{array}{l}\text { Li et al. } \\
\text { [47] }\end{array}$ & $\begin{array}{l}\text { Robust } \\
\text { tracking } \\
\text { control } \\
\text { problem in } \\
\text { NCS due to } \\
\text { networked } \\
\text { induced- } \\
\text { factors }\end{array}$ & $\begin{array}{l}\text { k-order } \\
\text { adaptive } \\
\text { discrete-time } \\
\text { sliding- mode } \\
\text { controller }\end{array}$ & $\begin{array}{l}\text { Improves } \\
\text { tracking } \\
\text { control } \\
\text { process in } \\
\text { NCS }\end{array}$ \\
\hline $\begin{array}{l}\text { Wu et al. } \\
\text { [48] }\end{array}$ & $\begin{array}{l}\text { Packet loss } \\
\text { problem in } \\
\text { discrete-time } \\
\text { NCS }\end{array}$ & $\begin{array}{l}\text { Dwell-time } \\
\text { mechanism }\end{array}$ & $\begin{array}{l}\text { Effective to } \\
\text { control } \\
\text { packet } \\
\text { drops in }\end{array}$ \\
\hline
\end{tabular}




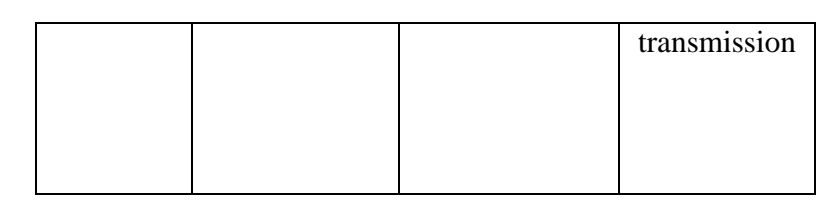

\begin{tabular}{|c|c|c|c|}
\hline Author & Problem & $\begin{array}{l}\text { Solution } \\
\text { Approach }\end{array}$ & Advantages \\
\hline $\begin{array}{l}\text { Almakhles et } \\
\text { al. [49] }\end{array}$ & $\begin{array}{l}\text { Quantization } \\
\text { noise, data } \\
\text { loss, } \\
\text { transmission } \\
\text { delay }\end{array}$ & $\begin{array}{l}\text { Adaptive } \\
\text { Quantizer } \\
\text { model with } \\
\text { sliding mode } \\
\text { controller }\end{array}$ & $\begin{array}{l}\text { Increases } \\
\text { network } \\
\text { system } \\
\text { performance }\end{array}$ \\
\hline Li et al. [50] & $\begin{array}{l}\text { Delay and } \\
\text { packet loss in } \\
\mathrm{T}-\mathrm{S} \text { fuzzy } \\
\text { systems }\end{array}$ & $\begin{array}{l}\text { stabilizing } \\
\text { controller }\end{array}$ & $\begin{array}{l}\text { Enhances } \\
\text { network } \\
\text { stability and } \\
\text { reduces the } \\
\text { packet loss } \\
\text { rate }\end{array}$ \\
\hline Tang et al. [51] & $\begin{array}{l}\text { Real-time } \\
\text { distribution } \\
\text { process }\end{array}$ & $\begin{array}{l}\text { Indicator } \\
\text { function and } \\
\text { advanced } \\
\text { Lyapunov- } \\
\text { Krasovskii } \\
\text { technique }\end{array}$ & $\begin{array}{l}\text { enhanced } \\
\text { stable } \\
\text { system }\end{array}$ \\
\hline Lu et al. [52] & $\begin{array}{l}\text { stability and } \\
\text { design } \\
\text { problem for } \\
\text { NCS }\end{array}$ & $\begin{array}{l}\text { quantizer \& } \\
\text { event based } \\
\text { triggered } \\
\text { model }\end{array}$ & $\begin{array}{l}\text { Improves } \\
\text { NCS } \\
\text { imperfection } \\
\text { and efficient } \\
\text { resource } \\
\text { utilization }\end{array}$ \\
\hline Tang et al. [53] & $\begin{array}{l}\text { Real-time } \\
\text { distribution } \\
\text { and } \\
\text { transmission } \\
\text { delay }\end{array}$ & $\begin{array}{l}\text { Fuzzy model } \\
\text { based joint } \\
\text { approach }\end{array}$ & $\begin{array}{l}\text { Enhanced } \\
\text { real-time } \\
\text { distribution } \\
\text { of input } \\
\text { delays }\end{array}$ \\
\hline Mo et al. [54] & $\begin{array}{l}\text { Packets } \\
\text { dropouts, } \\
\text { reliability } \\
\text { transmission } \\
\text { delay }\end{array}$ & $\begin{array}{l}\text { Novel } \\
\text { stochastic } \\
\text { model using a } \\
\text { linear discrete- } \\
\text { time technique }\end{array}$ & $\begin{array}{l}\text { Improve } \\
\text { network } \\
\text { reliability } \\
\text { and } \\
\text { performance }\end{array}$ \\
\hline $\begin{array}{l}\text { Wang and Liu } \\
\text { [55] }\end{array}$ & $\begin{array}{l}\text { Stability and } \\
\text { packet losing } \\
\text { at data } \\
\text { transmission }\end{array}$ & $\begin{array}{l}\text { Discrete-time } \\
\text { switched NCS } \\
\text { framework }\end{array}$ & $\begin{array}{l}\text { Achieves } \\
\text { efficiency to } \\
\text { avoid delay } \\
\text { and data } \\
\text { losing factor }\end{array}$ \\
\hline Sa et al. [56] & $\begin{array}{l}\text { Passive } \\
\text { attacks }\end{array}$ & $\begin{array}{l}\text { Random } \\
\text { switching } \\
\text { controller }\end{array}$ & $\begin{array}{l}\text { Effective for } \\
\text { resisting } \\
\text { some } \\
\text { passive } \\
\text { types } \\
\text { attacks }\end{array}$ \\
\hline Feng et al. [57] & Actuator fault & $\begin{array}{l}\text { Markov chain } \\
\text { and state } \\
\text { augmentation }\end{array}$ & $\begin{array}{l}\text { Resolves the } \\
\text { issue of } \\
\text { actuator } \\
\text { fault and }\end{array}$ \\
\hline
\end{tabular}

\begin{tabular}{|l|l|l|l|}
\hline & & method & $\begin{array}{l}\text { improves } \\
\text { the stability } \\
\text { of NCS }\end{array}$ \\
\hline Ming Li [58] & $\begin{array}{l}\text { The problem } \\
\text { caused by } \\
\text { delay and } \\
\text { data loss in } \\
\text { NCS }\end{array}$ & $\begin{array}{l}\text { Ethernet } \\
\text { Switched }\end{array}$ & $\begin{array}{l}\text { Minimizes } \\
\text { data loss } \\
\text { factor } \\
\text { during data } \\
\text { transmission }\end{array}$ \\
\hline Saha et al. [59] & $\begin{array}{l}\text { Controller } \\
\text { handling } \\
\text { problem }\end{array}$ & $\begin{array}{l}\text { Dynamic } \\
\text { scheduling } \\
\text { technique }\end{array}$ & $\begin{array}{l}\text { Reduces the } \\
\text { packet drop } \\
\text { rates }\end{array}$ \\
\hline
\end{tabular}

\begin{tabular}{|c|c|c|c|}
\hline Author & Problem & $\begin{array}{l}\text { Solution } \\
\text { Approach }\end{array}$ & Advantages \\
\hline $\begin{array}{l}\text { Zhiwen } \\
\text { and } \\
\text { Hongta } \\
\mathrm{o}[60]\end{array}$ & $\begin{array}{l}\text { Network } \\
\text { congestion } \\
\text { and Quality } \\
\text { degradation }\end{array}$ & $\begin{array}{l}\text { LQR (Linear- } \\
\text { Quadratic } \\
\text { Regulator)techniqu } \\
\text { e }\end{array}$ & $\begin{array}{l}\text { Enhances } \\
\text { QoS of } \\
\text { network and } \\
\text { achieves } \\
\text { effective } \\
\text { bandwidth } \\
\text { managemen } \\
\text { t }\end{array}$ \\
\hline $\begin{array}{l}\text { Yang et } \\
\text { al. [61] }\end{array}$ & $\begin{array}{l}\text { Actuator } \\
\text { control } \\
\text { process } \\
\text { under DoS } \\
\text { attack in } \\
\text { NCS }\end{array}$ & 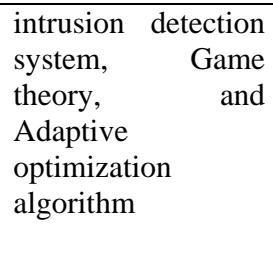 & $\begin{array}{l}\text { Resist DoS } \\
\text { attack and } \\
\text { improves } \\
\text { Actuator } \\
\text { control } \\
\text { process in } \\
\text { NCS }\end{array}$ \\
\hline $\begin{array}{l}\text { Li et al. } \\
\text { [62] }\end{array}$ & $\begin{array}{l}\text { Channel } \\
\text { access } \\
\text { issues }\end{array}$ & $\begin{array}{l}\text { Slotted } \\
\text { protocol, } \\
\text { assignment } \\
\text { technique, } \\
\text { convergent descent } \\
\text { optimization } \\
\text { algorithm }\end{array}$ & $\begin{array}{l}\text { Achieves } \\
\text { good } \\
\text { stability and } \\
\text { good packet } \\
\text { delivery } \\
\text { ratio }\end{array}$ \\
\hline $\begin{array}{l}\mathrm{Yu} \text { et } \\
\text { al. [63] }\end{array}$ & $\begin{array}{l}\text { closed-loop } \\
\text { convergenc } \\
\text { e problem }\end{array}$ & $\begin{array}{l}\text { quantization } \\
\text { scheme based on } \\
\text { Lyapunov- } \\
\text { krasovskii function }\end{array}$ & $\begin{array}{l}\text { Provides } \\
\text { better } \\
\text { convergenc } \\
\text { e property }\end{array}$ \\
\hline
\end{tabular}

\section{RESEARCH GAP}

On analyzing the above researches following research gaps were found in NCS.

- Most of the effective researches on NCS are evolving with the non-linear sampling rate, but these researches are only evolved with stability analysis of for a given scenario among consecutive sampling times. This can leads to conservative outcomes which can be overcome y using stochastic characterization for inter sampling times.

- Most of the researches were focused on determining the NCSs stability in which performance related issues were not much considered.

- The controller design for NCCs is less emphasized as most of the researchers initialize with a controller design by neglecting the challenges induced by NCS. 
- Most of the researches were focused on theoretical concepts of NCS and not much practical implementation where done.

- The security-related issues in NCS are rarely addressed in existing researches.

- Most of the researches have considered only the already existing research which means the same kind of perimeter selection for NCS design analysis.

Thus there is a need for a proper design NCS which can eliminate issues induced by NCS.

\section{STATE OF THE ART IN CURRENT RESEARCH}

This section idealizes the current researches held towards the Networked Control System (NCS) design. The numeric data for state of the art in the current work are extracted from the standard publications like IEEE, Elsevier, and Springer by providing "Networked Control System" as a keyword. The following Table.2, 3 and 4 indicates the numerical details of published manuscripts in IEEE, Springer, and Elsevier respectively (cited on 07-August-2018 (India: 4.30pm)).

Table 2: Numerical details from IEEE

\begin{tabular}{|l|l|}
\hline Type of publications (IEEE) & Total Counts \\
\hline Conferences & 155,941 \\
\hline Journals \& Magazines & 31,771 \\
\hline Early Access Articles & 1,400 \\
\hline Standards & 683 \\
\hline Books & 262 \\
\hline Courses & 204 \\
\hline
\end{tabular}

Table 3: Numerical details from Springer

\begin{tabular}{|l|l|}
\hline Type of publications (Springer) & Total Counts \\
\hline Series & 403 \\
\hline Journals \& Magazines & 718 \\
\hline Web pages & 183 \\
\hline Books & 61214 \\
\hline
\end{tabular}

Table 4: Numerical details from Elsevier

\begin{tabular}{|l|l|}
\hline Type of publications & Total Counts \\
\hline Web pages & 99 \\
\hline Journals \& Magazines & 42 \\
\hline Books & 1154 \\
\hline
\end{tabular}

The percentage wise analysis of the current research trend gives that the number of research manuscripts was presented in IEEE than Springer and Elsevier (as shown in Figure.2). The Springer has $100 \%, 63 \%, 3 \%$ of series, Web pages, and journals \& magazines manuscripts respectively are found in Springer. The IEEE publication has 100\%, 98\%, 100\%, $100 \%, 100 \%$ of conferences, journals \& magazines, early access articles, standards and courses for NCS respectively than Springer and Elsevier. The Elsevier has found less number of manuscripts published, i.e., $37 \%$ of web pages and
$3 \%$ of books. This indicates that still more research works are necessary to be performed.

\section{Statistics of Current research}

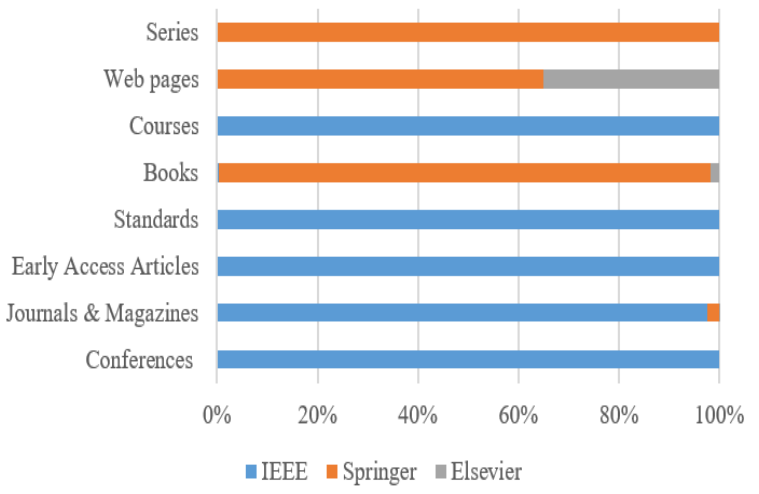

Figure 2: Analysis of the current research trend

\section{CONCLUSION}

This paper gives a detailed survey on networked control systems (NCSs) are discussed along with theoretical concepts of NCS, applications of NCS and issues of NCS. An overview of sampled data (SD) control, event-triggered control, and networked control is given along with the existing approaches. An extensive review of existing researches addressing various issues of NCS and proposing a solution by adopting different approaches is discussed. State of the art in current research over NCS is given by extracting publications details from IEEE, Springer, and Elsevier. From this, it is found that still more works need to perform on NCS by considering different performance parameters. Further, in analyzing these existing works, a research gap is addressed. From a research survey, various issues subjected to NCS were found which includes quantization, delay, non-linearity, data loss, etc.

This paper can be considered for further research in the domain of NCS. Control theory can be developed for distributed, packed based and asynchronous environment. This paper can be regarded as for stability enhancement of NCS under delay, packet dropping rate, etc., constraints. This paper can be considered for the development of real-time NCS.

\section{REFERENCES}

[1] AlthougHuang, Deqing, et al. "High-performance tracking of piezoelectric positioning stage using currentcycle iterative learning control with gain scheduling." IEEE Transactions on Industrial Electronics 61.2 (2014): 1085-1098.

[2] Qiu, Jianbin, et al. "Fuzzy-model-based reliable static output feedback $\mathrm{H} \infty$ control of nonlinear hyperbolic PDE systems." IEEE Trans. Fuzzy Syst 24.2 (2016): 388-400.

[3] Wang, Tong, Huijun Gao, and Jianbin Qiu. "A combined fault-tolerant and predictive control for network-based industrial processes." IEEE Transactions on Industrial Electronics 63.4 (2016): 2529-2536.

[4] D. Zhang, Q.-L. Han, and X. Jia, "Network-based output tracking control for a class of T-S fuzzy systems that can not be stabilized by nondelayed output feedback 
controllers," IEEE Trans. Cybern., vol. 45, no. 8, pp. 1511-1524, Aug. 2015.

[5] Zhang, L, Zhu, Y, Ning, Z. Resilient estimation for networked systems with variable communication capability. IEEE T Automat Control 2016; 61: 41504156.

[6] Lee, Tae H., Jianwei Xia, and Ju H. Park. "Networked control system with asynchronous samplings and quantizations in both transmission and receiving channels." Neurocomputing 237 (2017): 25-38.

[7] Pasand, Mohammad Mahdi Share, and Mohsen Montazeri. "Structural properties, LQG control and scheduling of a networked control system with bandwidth limitations and transmission delays." IEEE/CAA Journal of Automatica Sinica (2017).

[8] Cuenca, Angel, et al. "Periodic Event-Triggered Sampling and Dual-rate Control for a Wireless Networked Control System with Applications to UAVs." IEEE Transactions on Industrial Electronics (2018).

[9] Cuenca, A., et al. "A packet-based dual-rate PID control strategy for a slow-rate sensing Networked Control System." ISA transactions 76 (2018): 155-166.

[10] Borgers, D. P., et al. "Periodic event-triggered control of nonlinear systems using overapproximation techniques." Automatica 94 (2018): 81-87.

[11] J. Hespanha, P. Naghshtabrizi, and Y. Xu, "A survey of recent results in networked control systems," Proc. IEEE, vol. 95, no. 1, pp. 138-162, Jan. 2007

[12] G. Pratl, D. Dietrich, G. Hancke, and W. Penzhorn, "A new model for autonomous, networked control systems," IEEE Trans. Ind. Informat., vol. 3, no. 1, pp. 21-32, Feb. 2007.

[13] J. Lunze and D. Lehmann, "A state-feedback approach to event-based control," Automatica, vol. 46, no. 1, pp. 211-215, Jan. 2010.

[14] X. Wang and M. Lemmon, "Self-triggered feedback control systems with finite-gain stability," IEEE Trans. Autom. Control, vol. 45, no. 3, pp. 452-467, Mar. 2009.

[15] Y.-L. Wang and Q.-L. Han, "Modelling and controller design for discrete-time networked control systems with limited channels and data drift," Inf. Sci., vol. 269, pp. 332-348, Jun. 2014.

[16] Z. Du, D. Yue, and S. Hu, "Hळ stabilization for singular networked cascade control systems with state delay and disturbance," IEEE Trans. Ind. Informat., vol. 10, no. 2, pp. 882-894, May 2014.

[17] W.-A. Zhang and L. Yu, "A robust control approach to stabilization of networked control systems with timevarying delays," Automatica, vol. 45, no. 10, pp. 24402445, Oct. 2009

[18] M. Cloosterman, L. Hetel, N. van de Wouw, W. Heemels, J. Daafouz, and H. Nijmeijer, "Controller synthesis for networked control systems," Automatica, vol. 46, no. 10, pp. 1584-1594, Oct. 2010.

[19] Y. Mikheev, V. Sobolev, and E. Fridman, "Asymptotic analysis of digital control systems," Autom. Remote Control, vol. 49, pp. 1175-1180, 1988.
[20] Seuret, "A novel stability analysis of linear systems under asynchronous samplings," Automatica, vol. 48, no. 1, pp. 177-182, Jan. 2012.

[21] P. Naghshtabrizi, J. Hespanha, and A. Teel, "Exponential stability of impulsive systems with application to uncertain sampled-data systems," Syst. Control Lett., vol. 57, no. 5, pp. 378-385, May 2008.

[22] W. H. Chen and W. X. Zheng, "An improved stabilization method for sampled-data control systems with control packet loss," IEEE Trans. Autom. Control, vol. 57, no. 9, pp. 2378-2384, Sep. 2012.

[23] H. Gao, J. Wu, and P. Shi, "Robust sampled-data H1 control with stochastic sampling," Automatica, vol. 45, no. 7, pp. 1729-1736, Jul. 2009.

[24] D. Yue, Q.-L. Han, and J. Lam, "Network-based robust H1 control of systems with uncertainty," Automatica, vol. 41, no. 6, pp. 999-1007, Jun. 2005.

[25] P. Seiler and R. Sengupta, "An H1 approach to networked control, "IEEE Trans. Autom. Control, vol. 50, no. 3, pp. 356-364, Mar. 2005.

[26] W. A. Zhang and L. Yu, "Modelling and control of networked control systems with both network-induced delay and packet-dropout," Automatica, vol. 44, no. 12, pp. 3206-3210, Dec. 2008

[27] M. Donkers, W. Heemels, N. van de Wouw, and L. Hetel, "Stability analysis of networked control systems using a switched linear systems approach," IEEE Trans. Autom. Control, vol. 56, no. 9, pp. 2101-2115, Sep. 2011.

[28] L. Hetel, J. Daafouz, and C. Lung, "Analysis and control of LTI and switched systems in digital loops via an event-based modelling," Int. J. Control, vol. 81, no. 7, pp. 1125-1138, Jul. 2008.

[29] Kruszewski, W. Jiang, E. Fridman, J. P. Richard, and A. Toguyeni, "A switched system approach to exponential stabilization through communication network," IEEE Trans. Control Syst. Technol., vol. 20, no. 4, pp. 887 900, Apr. 2012

[30] H. Lin and P. Antsaklis, "Stability and persistent disturbance attenuation properties for a class of networked control systems: switched system approach,' Int. J. Control, vol. 78, no. 18, pp. 1447-1458, Dec. 2005.

[31] X. M. Sun, G.-P. Liu, W. Wang, and R. David, "Stability analysis for networked control systems based on average dwell time method," Int. J. Robust Nonlin. Control, vol. 20, no. 15, pp. 1774-1784, Oct. 2010.

[32] J. Nilsson, B. Bernhardsson, and B. Wittenmark, "Stochastic analysis and control of real-time systems with random time delays," Automatica, vol. 34, no. 1, pp. 57-64, Jan. 1998.

[33] F. Yang, Z. Wang, Y. S. Hung, and M. Gani, "H1 control for networked systems with random communication delays," IEEE Trans. Autom. Control, vol. 51, no. 3, pp. 511-518, Mar. 2006

[34] M. Donkers, W. Heemels, D. Bernardini, A. Bemporad, and V. Shneer, "Stability analysis of stochastic 
networked control systems," Automatica, vol. 48, no. 5, pp. 917-925, May. 2012.

[35] Tabbara, M., \& Nesic, D. (2008). Input-output stability of networked control systems with stochastic protocols and channels. IEEE Transactions on Automatic control, 53(5), 1160-1175.

[36] K. Arzen, "A simple event-based PID controller," in Proc. IFAC World Congress, Beijing, China, 1999.

[37] P. Tabuada, "Event-triggered real-time scheduling of stabilizing control tasks," IEEE Trans. Autom. Control, vol. 52, no. 9, pp. 1680-1685, Sep. 2007.

[38] J. Lunze and D. Lehmann, "A state-feedback approach to event-based control," Automatica, vol. 46, no. 1, pp. 211-215, Jan. 2010.

[39] X. Wang and M. Lemmon, "Self-triggered feedback control systems with finite-gain stability," IEEE Trans. Autom. Control, vol. 45, no. 3, pp. 452-467, Mar. 2009.

[40] Wu, Chengwei, et al. "Adaptive fuzzy control for nonlinear networked control systems." IEEE Trans. Syst. Man Cybern. Syst 47.8 (2017): 2420-2430.

[41] Xu, Hao, and Sarangapani Jagannathan. "Neural network-based finite horizon stochastic optimal control design for nonlinear networked control systems." IEEE transactions on neural networks and learning systems 26.3 (2015): 472-485.

[42] Qiu, Jianbin, Huijun Gao, and Steven X. Ding. "Recent advances on fuzzy-model-based nonlinear networked control systems: A survey." IEEE Transactions on Industrial Electronics 63.2 (2016): 1207-1217.

[43] Khanesar, Mojtaba Ahmadieh, et al. "Adaptive indirect fuzzy sliding mode controller for networked control systems subject to time-varying network-induced time delay." IEEE Transactions on Fuzzy Systems 23.1 (2015): 205-214.

[44] Yuan, Chengzhi, and Fen Wu. "Delay scheduled impulsive control for networked control systems." IEEE Transactions on Control of Network Systems 4.3 (2017): 587-597.

[45] Hamdy, Mohamed, Sameh Abd-Elhaleem, and M. A. Fkirin. "Time-Varying Delay Compensation for a Class of Nonlinear Control Systems Over Network via \$ $\mathrm{H}_{-}$ \{linfty\} \$ Adaptive Fuzzy Controller." IEEE Transactions on Systems, Man, and Cybernetics: Systems 47.8 (2017): 2114-2124.

[46] Razeghi-Jahromi, Mohammad, and Alireza Seyedi. "Stabilization of networked control systems with sparse observer-controller networks." IEEE Transactions on Automatic Control 60.6 (2015): 1686-1691.

[47] Li, Meng, and Yong Chen. "Robust Tracking Control of Networked Control Systems With Communication Constraints and External Disturbance." IEEE Trans. Industrial Electronics 64.5 (2017): 4037-4047.

[48] Wu, Di, et al. "On designing event-triggered schemes for networked control systems subject to one-step packet dropout." IEEE transactions on industrial informatics 12.3 (2016): 902-910
[49] Almakhles, Dhafer, et al. "An adaptive two-level quantizer for networked control systems." IEEE Transactions on Control Systems Technology 25.3 (2017): 1084-1091.

[50] Li, Hongbo, et al. "Stabilization and Separation Principle of Networked Control Systems Using the TS Fuzzy Model Approach." IEEE Trans. Fuzzy Systems 23.5 (2015): 1832-1843.

[51] Tang, Bin, Jun Wang, and Yun Zhang. "A delaydistribution approach to stabilization of networked control systems." IEEE Transactions on Control of Network Systems 2.4 (2015): 382-392.

[52] Lu, Zhong-Da, et al. "Event-Triggered H \$linfty \$ Fuzzy Filtering for Networked Control Systems With Quantization and Delays." IEEE Access 6 (2018): 2023120241.

[53] Tang, Bin, Shiguo Peng, and Yun Zhang. "Fuzzy-ModelBased Robust $\mathrm{H} \infty$ Design of Nonlinear Packetized Networked Control Systems." IEEE Trans. Fuzzy Systems 24.3 (2016): 544-557.

[54] Mo, Huadong, et al. "Modeling and analysis of the reliability of digital networked control systems considering networked degradations." IEEE Transactions on Automation Science and Engineering 14.3 (2017): 1491-1503.

[55] Wang, Jufeng, and Chunfeng Liu. "Exponential stability of a class of networked control systems with disturbed controllers." Advances in Difference Equations 2016.1 (2016): 5

[56] de Sá, Alan O., Luiz FR da Costa Carmo, and Raphael CS Machado. "A controller design for mitigation of passive system identification attacks in networked control systems." Journal of Internet Services and Applications 9.1 (2018): 2

[57] Yan-feng, Wang, et al. "Fault-tolerant control for networked control systems with limited information in case of actuator fault." Mathematical Problems in Engineering 2015 (2015).

[58] Li, Ming. "Delay analysis of networked control systems based on $100 \mathrm{M}$ switched Ethernet." The Scientific World Journal2014 (2014).

[59] Saha, Indranil, Sanjoy Baruah, and Rupak Majumdar. "Dynamic scheduling for networked control systems." Proceedings of the 18th International Conference on Hybrid Systems: Computation and Control. ACM, 2015.

[60] Zhiwen, Wang, and Sun Hongtao. "Control and optimization of network in networked control system." Mathematical Problems in Engineering 2014 (2014).

[61] Yang, Hongjiu, et al. "Adaptive dynamic programming for security of networked control systems with actuator saturation." Information Sciences (2018).

[62] Li, Zhihui, Ge Guo, and Zhongchang Liu. "Communication parameter design for networked control systems with the slotted ALOHA access protocol." Information Sciences 447 (2018): 205-215. 
Communications on Applied Electronics (CAE) - ISSN : 2394-4714

Foundation of Computer Science FCS, New York, USA

Volume 7-No. 20, September 2018-www.caeaccess.org

[63] Wang, Yilin, and Shuanghe Yu. "An improved dynamic quantization scheme for uncertain linear networked control systems." Automatica 92 (2018): 244-248. 